\title{
Graphene quantum dot on boron nitride: Dirac cone replica and Hofstadter butterfly
}

\author{
L.A. Chizhova, F. Libisch ** and J. Burgdörfer \\ Institute for Theoretical Physics, Vienna University of Technology, \\ Wiedner Hauptstraße 8-10, 1040 Vienna, Austria, EU
}

(Dated: December 8, 2018)

\begin{abstract}
Graphene flakes placed on hexagonal boron nitride feature in the presence of a magnetic field a complex electronic structure due to a hexagonal moiré potential resulting from the van der Waals interaction with the substrate. The slight lattice mismatch gives rise to a periodic supercell potential. Zone folding is expected to create replica of the original Dirac cone and Hofstadter butterflies. Our large-scale tight binding simulation reveals an unexpected coexistence of a relativistic and nonrelativistic Landau level structure. The presence of the zeroth Landau level and its associated butterfly is shown to be the unambiguous signature for the occurrence of Dirac cone replica.
\end{abstract}

PACS numbers: 73.22.Pr, 71.70.Di, 81.05.ue, 71.70.-d

\section{INTRODUCTION}

The electronic and transport properties of graphene strongly depend on the substrate graphene is placed on or substituted in. For instance, in the case of widely-used $\mathrm{SiO}_{2}$, the roughness of the substrate surface introduces a corrugation of the graphene monolayer,,${ }^{1}$ puddles, ${ }^{2}$ and charge traps ${ }^{[3}$ Reducing substrate-induced disorder is critical for achieving higher carrier mobility especially in transport applications. Graphene on clean transition metal surfaces [e.g., iridium (Ir) ${ }^{4}$ or ruthenium ${ }^{5}$ ] or on graphite ${ }^{\sqrt{6}}$ have been shown to feature much reduced disorder ${ }^{788}$ More recently, the wide gap insulator hBN received major attention as substrate material since it is inert to the carriers in graphene near the Fermi energy $[9110$

A small lattice mismatch between graphene and the substrate leads to periodic potential modulations on a scale much larger than the lattice vector ${ }^{[11] 13}$ Such a potential is formed, for instance, by a grid of electron-beam deposited adatoms on graphene, $e^{1114}$ by the misalignment of graphene layers in twisted bilayer graphene, $\frac{15 / 16}{16}$ or by a small lattice mismatch between graphene and a hexagonal substrate (BN or Ir), resulting in a so-called moiré pattern. ${ }^{910}$ For hexagonal boron nitride, the layersubstrate interaction is of Van der Waals type. The effect of the substrate can be, to first order, captured by an additional smooth periodic potential with superlattice periodicity $a^{S}$ large compared to the lattice periodicity $a$. For graphene on hBN and an alignment angle $\phi=0^{\circ}$, $a^{S}=13.8 \mathrm{~nm}\left(a^{S} / a \gtrsim 50\right)$. Introducing the additional length scale $a^{S}$ into the physics of graphene devices gives rise to interesting new phenomena. The zone folding due to the periodic superlattice leads to additional high symmetry points in the bandstructure ${ }^{111}$ DFT simulations of a supercell of graphene on hBN and STM measurements suggest additional Dirac cones in the bandstructure $\frac{12}{12}$ Indeed, recent experiments of magnetotransport 17 and quantum capacitance effects in a magnetic field ${ }^{[18}$ observe the formation of replica of Landau level structures energetically above and below the primary structure associated with the "main" Dirac point. These structures were attributed $\sqrt{17}$ to satellite Dirac-cones caused by the
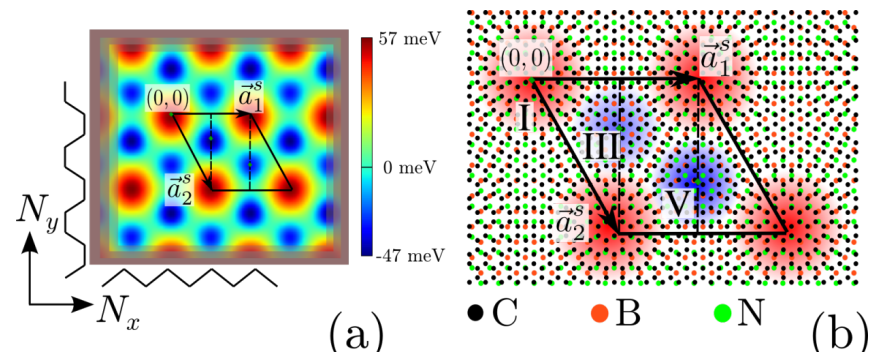

FIG. 1. (a) Schematic view of graphene flake with the superlattice potential landscape, (b) superlattice unit cell for graphene on BN with carbon (black), boron (orange) and nitrogen (green) atoms. The areas $I$ (red), III and $V$ (blue) are taken from Ref. 20]

moiré pattern of the superlattice. However, a zeroth Landau level at the satellite, the hallmark of Dirac-like Landau level structures $\frac{19}{19}$ is conspicuously missing. 17118 In the present work, we aim at elucidating the origin of these satellites, and explaining why experiments have, up to now, failed to reproduce the expected zeroth Landau level in the satellite structures.

Based on recent ab-initio DFT calculations, 2021 we simulate a realistic, extended graphene nanoflake interacting with an hBN substrate (Fig. 1). We benchmark our description by reproducing characteristic features of graphene on hBN in a magnetic field, e.g., the Hofstadter butterfyy $\frac{18 \sqrt{122} \mid 24}{24}$ as well as the observed satellite structures. Our results suggest that these satellites are caused by parabolic extremal points in the bandstructure giving rise to Schrödinger-like Landau levels rather than replica of Dirac cones. Key is the observation that, coincidentally, both Dirac- and Schrödinger like dispersion relations give rise to linear Landau level structures when plotted as a function of the back gate voltage. The distinguishing feature turns out to be the presence (or absence) of a magnetic-field independent zeroth Landau level. Only when employing an unrealistically strong superlattice potential true Dirac-cone like satellite structures, that include a zeroth Landau level, emerge close to the main Dirac point. 


\section{GRAPHENE MODEL HAMILTONIAN}

While our numerical simulation employs a third-order tight-binding Hamiltonian (for details see Ref. 25) realistically reproducing the bandstructure of graphene, it is instructive for the discussion of superlattice effects to briefly recall the essential features of the continuous lowenergy approximation in terms of an effective massless Dirac Hamiltonian with the spinor components related to the sublattice degree of freedom, $\left(\psi_{A}, \psi_{B}\right)$, the so-called pseudospin. $\sqrt[3 \mid 26]{ }$ Including the physical spin, one arrives at a four-component spinor.25 In the presence of a homogeneous magnetic field $\vec{B}=\vec{\nabla} \times \vec{A}$ perpendicular to the graphene plane as well as an external electrostatic potential the Dirac Hamiltonian reads

$$
\begin{aligned}
H_{D}= & v_{F} \vec{\sigma} \cdot\left(\hat{\vec{p}}-\frac{e}{c} \vec{A}\right) \otimes \tau_{1}+v_{F} \overrightarrow{\sigma^{*}} \cdot\left(\hat{\vec{p}}-\frac{e}{c} \vec{A}\right) \otimes \tau_{2} \\
& +V(\vec{r}) \cdot \sigma_{0} \otimes \tau_{0}+W(\vec{r}) \cdot \sigma_{z} \otimes \tau_{0}
\end{aligned}
$$

where the Pauli matrices $\sigma_{x, y, z}\left(\tau_{x, y, z}\right)$ and unit matrix $\sigma_{0}\left(\tau_{0}\right)$ act on the $A-B$ sublattice $\left(K-K^{\prime}\right.$ or valley) degree of freedom, $\tau_{1,2}=\left(\tau_{0} \pm \tau_{z}\right) / 2, e$ is the elementary charge and $c$ is the speed of light. In Eq. (1) we have distinguished two different classes of potentials: the (slowly varying) background potential $V(\vec{r})$ (represented by the unit matrix in sublattice space) breaking the particle-hole symmetry within the Dirac double cone and the (short range) contribution $W(\vec{r})$ (represented by $\sigma_{z}$ in sublattice space) breaking sublattice symmetry. $W(\vec{r})$ effectively introduces a finite "mass" for the Dirac fermions and thus a band gap at the Dirac point.27

The effect of the hBN substrate can now be modeled by judicious choices for the potentials $V(\vec{r})$ and $W(\vec{r})$. The moiré pattern of graphene on hBN with an alignment angle of $\phi \approx 0^{\circ}$ features a period of $a^{S}=13.8$ $\mathrm{nm}$ and hexagonal superlattice vectors $\vec{a}_{1}^{S}=(13.8,0) \mathrm{nm}$ and $\vec{a}_{2}^{S}=(6.9,11.9) \mathrm{nm}$ [see Fig. 1(b)]. The supercell may be partitioned into five regions based on the relative local alignment of the graphene and hBN layer [see labels in Fig. 1(b) and Ref. 20]: in region $I$, the carbon atoms of one sublattice $A$ are on top of boron and the carbon atoms of the other sublattice $B$ on top of nitrogen; the region $I I I$ features the carbon atoms of $A$ on top of the nitrogen atoms and the atoms of $B$ are in the middle off a BN hexagon while in region $V$ the carbon atoms of $A$ are on top of boron atoms while now the $B$ atoms are located off the $\mathrm{BN}$ hexagons. We deduce realistic potential parameters from recent ab-initio DFT calculations, $20 \mid 21$ where sublattice symmetry breaking potentials of type $W(\vec{r})$ feature broad maxima and minima at the centers of the regions $I, I I I$ and $V$, while the transition regions $I I$ and $I V$ feature intermediate stacking configurations and potential values. We thus expand $W(\vec{r})$ in Gaussians according to

$$
W(\vec{r})=\sum_{i=I, I I I, V} W_{i} \exp \left(-\frac{\left(\vec{r}-\vec{R}_{i}\right)^{2}}{2 w_{i}^{2}}\right)
$$

with amplitudes $W_{I}=57 \mathrm{meV}, W_{I I I}=-34 \mathrm{meV}$, $W_{V}=-47 \mathrm{meV}$ taken from Sachs et al., 20 and widths $0.63 \cdot w_{I}=w_{I I I}=w_{V}=7 \mathrm{~nm}$ from geometrical considerations. We note that local doping by, e.g., charge traps may lead to further local potential variation in the experiment. While the potential $W(\vec{r})$ opens a gap near the Dirac point, the substrate interaction represented by the potential $V(\vec{r})$ breaks the electron-hole symmetry of the Dirac Hamiltonian (Eq. 1). Note that in the numerical solution employing a third-order TB Hamiltonian (see below) the exact particle-hole symmetry is already weakly broken in the absence of $V$. The experimental data, indeed, reveals a pronounced asymmetry between the electron and hole satellites. ${ }^{17}$ DFT calculations of the adhesion energy of graphene on hBN suggest only $20 \mathrm{meV}$ stronger binding in region $\mathrm{V}$ than in other regions.20 As we have verified numerically, this estimate is too small to reproduce the experimentally observed asymmetry. Yankowitz et al ${ }^{12}$ estimate the variation of $V(\vec{r})$ from second-order perturbation theory to be of the order of $120 \mathrm{meV}$. We expand $V(\mathbf{r})$ in terms of Gaussians of the form of Eq. (2), with the amplitudes of $V_{V} \approx-100$ $\mathrm{meV}, V_{I}=V_{I I I}=0$, placing the potential minimum at the site of the strongest adhesion.

The experiment indicates the complete lifting of the four-fold degeneracy of the zeroth Landau level. In addition to the Zeeman splitting of the spin degree of freedom with $\Delta E_{Z}=g_{\text {eff }} \mu_{B} B$ also the valley degeneracy is lifted by exchange interaction related to the energy cost of a spin reversal relative to adjacent (polarized) spins. 28 Measurements of quantum Hall states as a function of magnetic field suggest a linear increase of valley splitting with magnetic field, which is, to our knowledge, currently not fully understood theoretically! 28 To account for such a many-body (MB) effect within our single-particle description, we add to the potential $W(\vec{r})$ a phenomenological correction

$$
W_{\mathrm{MB}}(\vec{r})=\alpha \cdot B e^{-r^{2} / 2 w_{I}^{2}},
$$

that scales linearly in $B$ with $\alpha=8 \mathrm{meV} / \mathrm{T}$ taken from experiment 28 The spin coupling also enhances the gyromagnetic ratio $g_{\text {eff }}$ governing the Zeeman effect relative to its bare value $g_{0}=2$.

To elucidate the evolution of the spectrum of a graphene flake in the presence of a superlattice potential, we first consider ideal, free-standing graphene in the Dirac approximation [Eq. (1)], with $V=W=0$. In the presence of a perpendicular magnetic field one obtains the Landau level spectrum for Dirac fermions 19

$$
E_{n}^{D}(B)=\operatorname{sgn}(n) \sqrt{2|e| \hbar v_{F}^{2}|n| B}, \quad n \in Z_{0},
$$

This spectrum has three prominent features: (i) the existence of a $0^{\text {th }}$ Landau level $(n=0)$, which does not depend on the magnetic field, (ii) a graphene-specific degeneracy of the Landau levels due to the valley symmetry. and (iii) a square root dependence of all $n \neq 0$ Landau levels on the magnetic field. This non-equidistant spacing 


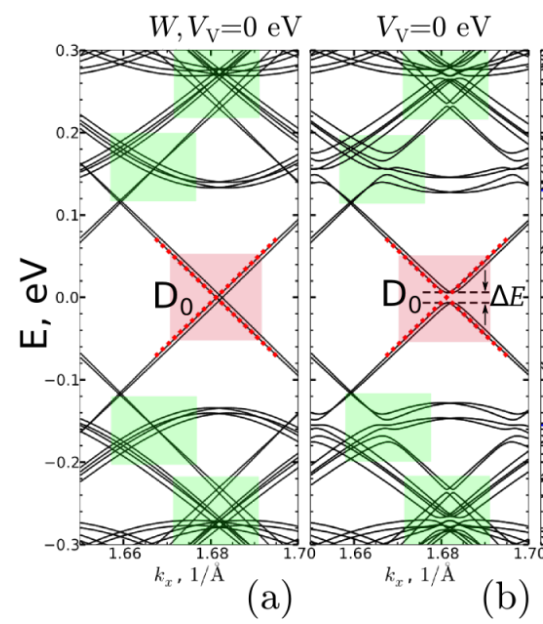

(a)

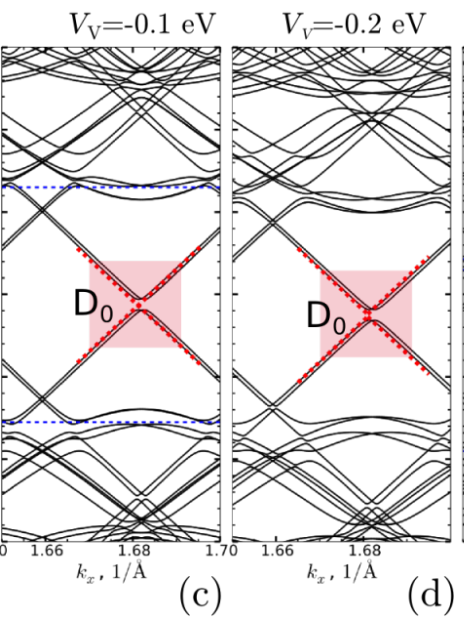

(d)

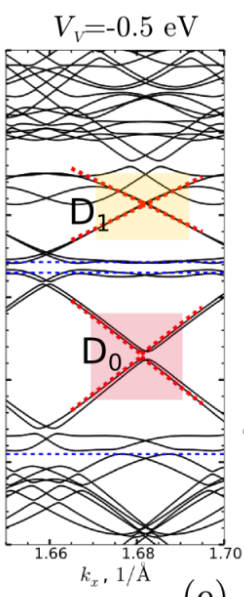

(e)

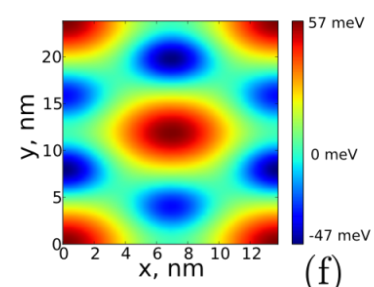

(f)

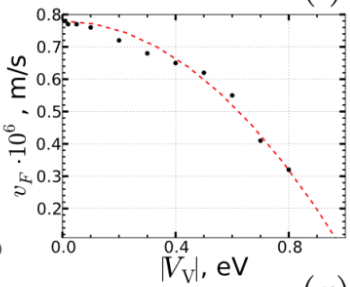

$(\mathrm{g})$

FIG. 2. Cut through bandstructure $\left(k_{y}=0\right)$ of bulk graphene with periodic superlattice potential within the reduced zone structure of the superlattice calculated using third nearest-neighbor tight-binding model: (a) $V=W=0$ eV, i.e. free-standing graphene, (b)-(e) $W_{I}=57 \mathrm{meV}, W_{I I I}=-34 \mathrm{meV}$ and $W_{V}=-47 \mathrm{meV}$ with varying $V_{V}$. The red dashed lines show a linear fit to the main Dirac cone $\mathrm{D}_{0}$ (red regions) and to the secondary cone $\mathrm{D}_{1}$ (yellow area) in (e), from which values of the Fermi velocity $v_{F}$ are extracted. Green regions in (b) shows the formation of avoided crossings due to the moire potential, which are absent in (a). (f) Potential landscape of the supercell used in the bandstructure calculations. (g) The dependence of the Fermi velocity $v_{F}$ of $\mathrm{D}_{0}$ on the amplitude of the superlattice potential $V_{V}$.

provides a clear-cut distinction to the equidistant level spacing of non-relativistic Schrödinger electrons where the Landau level spectrum takes on the form of a harmonic oscillator ${ }^{29} E_{n}^{S}(B)=\hbar \omega_{B}(n+1 / 2)$.

In current experiments, however, the relation between magnetic field and the back gate voltage $\left(V_{\mathrm{BG}}\right)$ rather than the energy $E$ is accessible. It is therefore instructive to inquire into the mapping of Dirac-like and Schrödinger-like Landau levels onto the $V_{\mathrm{BG}}-B$ plane. Applying a back-gate voltage $V_{\mathrm{BG}}$ induces a charge $Q$ (proportional to $V_{\mathrm{BG}}$ ) on the graphene flake by capacitive coupling. This in turn changes the Fermi level of the graphene flake. A capacitive coupling model predicts, due to the linear density of states (DOS) of Dirac electrons, a square-root relation ${ }^{30}$ between $V_{\mathrm{BG}}$ and $E$, $E \propto \sqrt{V_{\mathrm{BG}}}$. Therefore, Eq. (4) predicts a linear $V_{\mathrm{BG}^{-}}$ $B$ relation. Remarkably, the same holds for the nonrelativistic Schrödinger electrons since the nonrelativistic density of states in $2 D$ is energy independent, $\rho_{S}=$ const, and $Q \sim V_{\mathrm{BG}} \sim E$. Consequently, the linear relation between $V_{\mathrm{BG}}$ and $B$ or, equivalently, $V_{\mathrm{BG}}$ and the charge carrier number $N$ is found for both a Dirac-like and Schrödinger-like spectrum and cannot be used to reliably identify a Dirac cone or its replica. Instead, the distinctive feature is therefore the presence or absence of the $n=0$ level.

Another closely related and remarkable feature is the Hofstadter butterfly, $18 / 22[24$ observed in the simultaneous presence of the spatially periodic superlattice and the $2 \pi$ periodic magnetic phase characterized by the magnetic flux through the area $A$ of one unit cell of the superlattice, ${ }^{22 \mid 2431} \phi / \phi_{0}=B A e / h$. Because of the large lattice constant of the supercell, the Hofstadter butterfly becomes accessible at moderate laboratory field strength of the $B$ field.

\section{SIMULATIONS}

We simulate the response of a finite-sized patch of graphene with dimensions $N_{x} \times N_{y}=170 \times 140 \mathrm{~nm}^{2}$ (see Fig. 1(a)), surrounded by a repulsive edge potential to eliminate edge effects, using a third-nearest neighbor tight-binding approach as outlined in Ref. 25. We include the hBN substrate and many-particle effects through the potentials of Eqs.(2)-(3) discussed above, the magnetic field by a Peierls phase factor ${ }^{32}$, and account for the Zeeman term by first-order perturbation theory.

We first consider the unperturbed [Fig. 2(a)] and the superlattice-induced bandstructure [Fig. [2(b)-(e)]. To calculate the bandstructure we use a supercell with the potential distribution depicted in Fig. 2(f) imposing periodic boundary conditions. The unperturbed band structure $(V=W=0)$ is displayed within the first Brillioun zone of the reciprocal supercell. The parabolic bands [Fig. 2(a)] correspond to cuts through back-folded offcenter $\left(k_{y} \neq 0\right)$ cones while the near linear bands correspond to the main cone $\mathrm{D}_{0}$ and its replica centered at $k_{y}=0$. In the presence of the substrate interactions $W(\vec{r})$, the band gap $\Delta E$ for the main Dirac cone is $\Delta E=10 \mathrm{meV}$, consistent with the experiment ${ }^{[18}$ Moreover, the presence of $W$ induces numerous avoided crossings [green areas in Fig. 2(b)] which are absent in freestanding graphene [green areas in Fig. 2(a)]. Adding the on-site potential $V(\mathbf{r})$ further enhances the particle-hole asymmetry (beyond the small assymetry of the graphene 

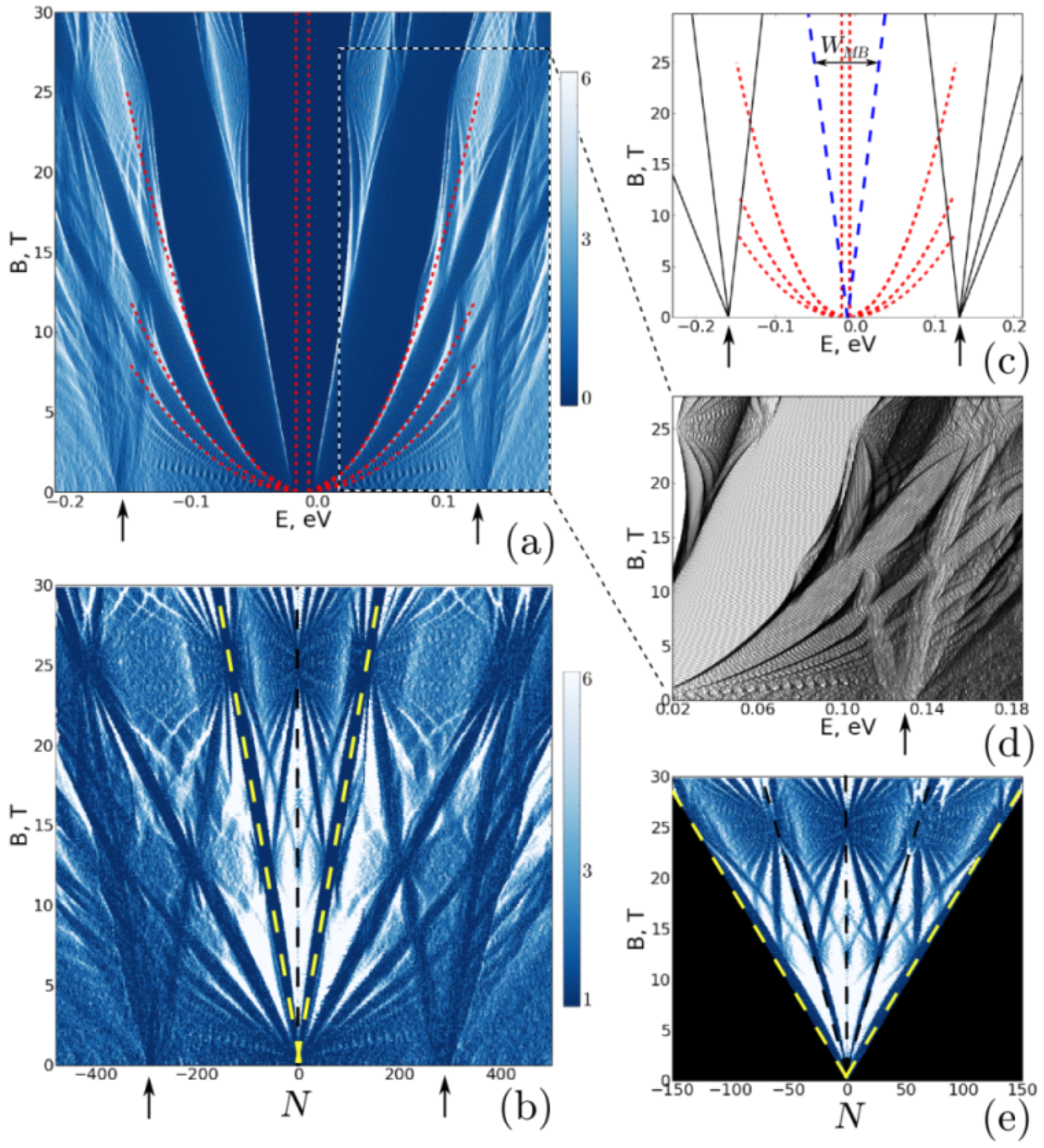

FIG. 3. Density of states of a graphene flake in the presence of a superlattice potential $\left(V_{V}=-0.1 \mathrm{eV}\right)$ : (a) as a function of electron energy and magnetic field; (b) same as (a) however as a function of the number of charge carriers $N$; (c) schematic plot of the important Landau level structures seen in (a): red dashed lines represent the Landau levels of the main Dirac cone including the energy gap $\Delta E=10 \mathrm{meV}$ [see Fig. 2]; blue dashed lines denote the splitting of the zeroth Landau level due to $W_{\mathrm{MB}}$; solid lines represent the Landau levels of the two Schrödinger-like satellites, whose origin is marked with black arrows in (a)-(d). (d) magnification of the area with the right satellite structure in (a). (e) Four-fold splitting of the $0^{\text {th }}$ Landau level including $W_{\mathrm{MB}}$ and Zeeman term with an enhanced $g=5$ due to electron-electron interaction [compare the same parameter region confined by yellow dashed lines in (b), where the Zeeman term is not included]. 

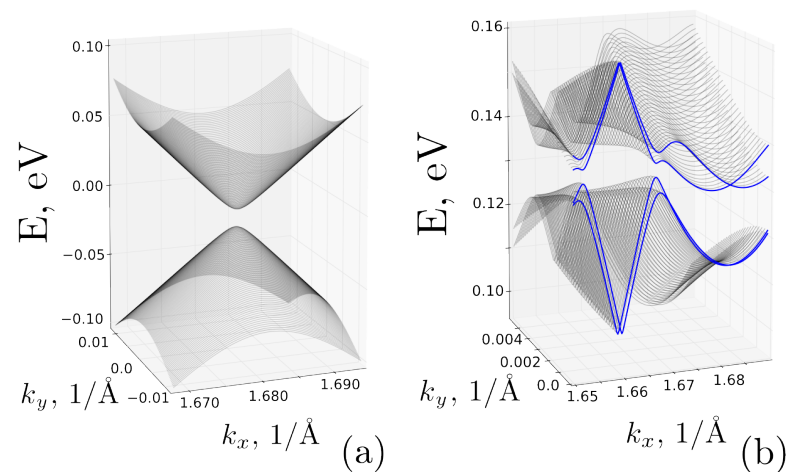

FIG. 4. Two-dimensional zero-field $(B=0)$ bandstructure $E\left(k_{x}\right)$. (a) Near the main Dirac cone $\mathrm{D}_{0}$ (see Fig 2(c)). (b) Bandstructure in the region of the satellite structure marked by the right arrow in Fig 3 (a) and (c).
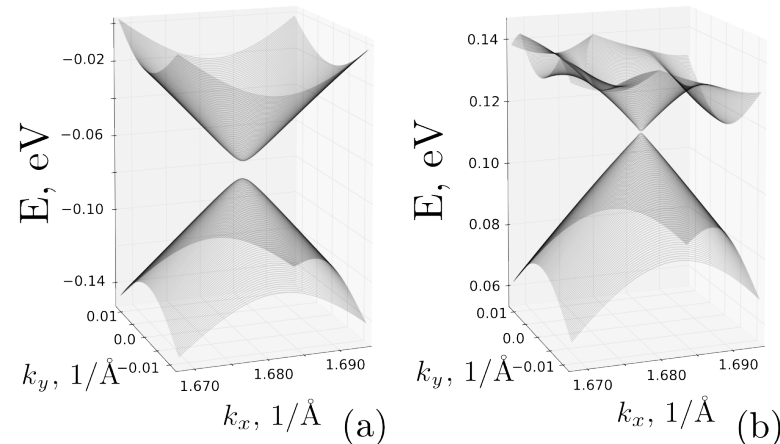

FIG. 5. Two-dimensional band structure for graphene on hBN with an unrealistically enhanced superlattice potential $\left(V_{V}=-0.5 \mathrm{eV}\right)$. (a) Primary Dirac cone $\mathrm{D}_{0}$, (b) secondary Dirac cone $\mathrm{D}_{1}$ (see Fig[2(e))

bandstructure as captured by the third nearest-nearest neighbor approximation ${ }^{33}$ ), shifts the position of the main cone $\mathrm{D}_{0}$ relative to $E=0 \mathrm{eV}$ by (to first order) the average of the superlattice potential [Fig. 2(c)-(e) red area] and enhances the size of the avoided crossings. Furthermore, the effective Fermi velocity $v_{F}$ of the main Dirac cone [Fig. 2(a)-(e) red area] decreases with increasing amplitude $V_{V}$ [Fig. 2( $\left.(\mathrm{g})\right]$. In the case of free-standing graphene the Fermi velocity is $v_{F 0}=0.78 \cdot 10^{6} \mathrm{~m} / \mathrm{s}$. With increasing $\left|V_{V}\right|$ the velocity $v_{F}$ drops proportionally to the square of the amplitude of the on-site potential $V_{V}$, in agreement with second-order perturbation theory, 34 which predicts a velocity renormalization

$$
v_{F \vec{k}}=v_{F 0}-\sum_{\vec{G} \neq 0} \frac{2|V(\vec{G})|^{2}}{v_{F 0}|\vec{G}|} \sin ^{2} \theta_{\vec{k}, \vec{G}} .
$$

$\vec{G}$ is the reciprocal lattice vector, $V(\vec{G})$ is the Fourier transform of the superlattice potential $V(\vec{r})$ of the form of Eq. 22 , and $\theta_{\vec{k}, \vec{G}}$ is the angle between $\vec{k}$ and $\vec{G}$. For a finite alignment angle $\phi$ between the hBN layer and the graphene, the periodicity $a^{S}$ of the moiré pattern is decreased, thereby increasing the size of the Billouin zone in reciprocal space. Consequently, the additional bands due to the overlap between the main Dirac cone and its replica lie further away from the Dirac point, as we have verified numerically.

We present the DOS for a realistic value of $V_{V}=-0.1$ $\mathrm{eV}$ in both the $E-B$ plane [Fig. 3(a,d)] and the $N-B$ plane [Fig. 3(b)] where $N$ is the number of charge carriers in the quantum dot $\left(\propto V_{\mathrm{BG}}\right.$ within a linear capacitance model). For the transformation from energy to charge carrier number we do not explicitly use the square root dependence $E \sim \sqrt{N}$ for bulk graphene but sum over the number of eigenstates (or charge) of the finite size flake lying between the Dirac cone and the appropriate Fermi energy $E_{F}$, to accurately account for deviations from the linear DOS close to the Dirac point.

The calculated DOS displays the formation of Landau levels emanating from the Dirac point of the main cone $\mathrm{D}_{0}$ [Fig. 3(a,c)]. The parabolic dependence [Eq. [4] ] $B \sim E^{2}$ [see red dashed parabolas in Fig. 3(a) and the schematic plot in (c)] confirms a relativistic diamagnetic response behavior. Moreover, the curvature of the parabolas determined by the Fermi velocity $v_{F}=$ $0.76 \cdot 10^{6} \mathrm{~m} / \mathrm{s}$ [Eq. (4)] extracted from the fit to the main Dirac cone in the bandstructure [red dashed lines in Fig.2(c)] agrees well with that of the simulated DOS. The zeroth Landau level splits linearly due to the many-body correction [Eq. (3)]. As discussed in Sec. II, the Landau levels as a function of $V_{\mathrm{BG}}$ (or here as a function of charge carrier number $N$ ) increase linearly with the magnetic field $B$ [see Fig. 3(b)] in agreement with experiment [see, for example, Refs. 17 and [18. Our numerical data also reproduce the pronounced splitting of the four-fold degeneracy of the zeroth Landau level [Fig. 3(e)] when including the B-field dependent many-body term $W_{\mathrm{MB}}$ and the Zeeman term with strongly enhanced gyromagnetic ratic ${ }^{28} g_{\text {eff }}=5$. Note the effective $g$ factor will, in general, be different for different Landau levels. Our effective many-body potential $W_{\mathrm{MB}}$ has negligible influence on energies far away from the Dirac point, e.g., on the satellite structures. It should be noted that our simulation does not reproduce a gap at the Dirac point as function of $N$ seen in the experiment [see Fig. 3(c)]. The latter results from quantum capacitance effects ${ }^{30}$ not included in our simulation.

Superlattice-induced effects on the diamagnetic spectrum are prominently visible: we observe a Hofstadter spectrum with "diamond"-like structures ${ }^{22}$ which are most pronounced at rational fractions 22131 of $\phi / \phi_{0}$. For the hexagonal superlattice with a period of $a^{S}=13.8 \mathrm{~nm}$, this ratio equals one at $B_{0}=25.5 \mathrm{~T}$ [see Fig. 3 (a, d)]. Moreover, we observe two distinct satellite features which evolve (approximately) linearly in the $E-B$ plane emerging at $B=0$ near $E=0.13 \mathrm{eV}$ and $-0.16 \mathrm{eV}$ [marked by arrows in Fig. 3(a, c, d)]. These satellite structures clearly display a non-relativistic rather than a relativistic $E(B)$ dependence. A closer look into the $B=0$ band- 

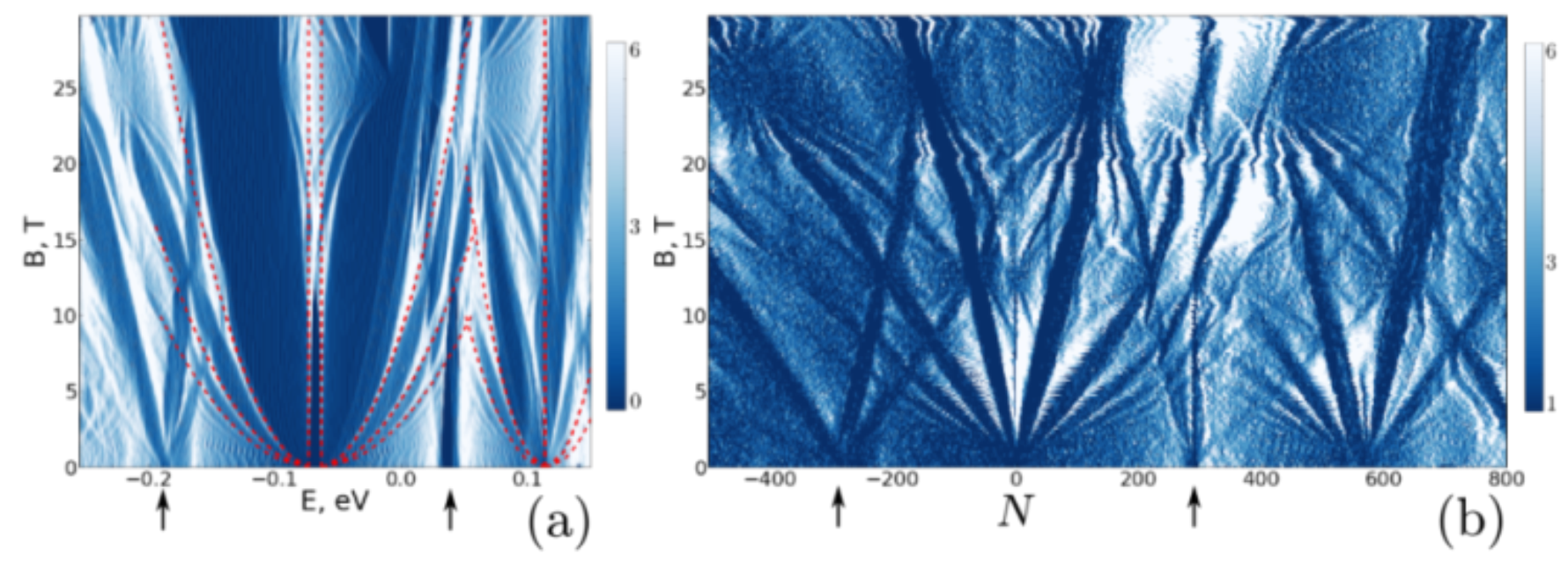

FIG. 6. Density of states of a graphene flake on $\mathrm{hBN}$ however with an unrealistically large superlattice potential $\left(V_{V}=-0.5 \mathrm{eV}\right.$ and $\left.W_{\mathrm{MB}}=0\right)$ : (a) in the $B$ - $E$ plane, as a function of magnetic field and electron energy; (b) in the $B-N$ plane, as a function of magnetic field and number of charge carriers. Black arrows at $B=0$ mark the same Schrödinger like satellites as in Fig. 3

structure [Fig. 2(c)] reveals that they originate from a region with a parabolic rather than a linear $E(k)$ dispersion. At these energies, the $2 \mathrm{D}$ bandstructure $E\left(k_{x}, k_{y}\right)$ [Fig. 4 near the satellites does not show cone-like structures unlike near the main Dirac cone. Consequently, the Landau levels show a Schrödinger-like rather than a Dirac-like [Eq. (4)] B-field dependence as a function of $E_{F}$. However, when plotted as a function of the charge carrier number $N$ or, equivalently, as a function of $V_{\mathrm{BG}}$ a linear $B-N\left(B-V_{\mathrm{BG}}\right)$ dependence emerges [Fig. 3(b)] for both the main Dirac cone and the satellites and this discriminating feature is lost. Such a linear dependence was seen in the experiment for the satellites $\frac{17118}{17}$ and was attributed to a Dirac cone replica. It is the absence of the $0^{\text {th }}$ Landau level and its Hofstadter butterfly for the satellites both in experiment and in our simulation that unambiguously confirms that the satellites are associated with a parabolic band structure rather than with a Dirac cone. We note that, strictly speaking, no real cone structures appear in the $2 \mathrm{D}$ bandstructure at the energy of the satellite states for the present moiré potential. All conelike dispersions visible in a $1 \mathrm{D}$ cut at $k_{y}=0$ show, upon consideration of the full 2D bandstructure, only avoided crossings [Fig. 4(b)], and not a true Dirac cone [Fig. 4(a)].

The origin of the non-relativistic dispersion can be easily traced to the unperturbed spectrum of bulk graphene [Fig. 2(a)]. The satellites emerge from parabolic bands with energies $|\Delta E| \approx 0.15 \mathrm{eV}$ above and below the Dirac points. Replica $\mathrm{D}_{1}$ of the Dirac cone centered at $k_{y}=0$ appear at much higher energies $|\Delta E| \gtrsim 0.28 \mathrm{eV}$ and are submerged in a region of high DOS. Accordingly, the superlattice potential resulting from the van der Waals interaction with the hBN substrate, which is of the order $|V| \lesssim 0.1 \mathrm{eV}$, represents only a moderately weak perturbation of the parabolic bands giving rise to distortion and narrow avoided crossings but cannot significantly shift the distant Dirac cone into the region of low DOS and into the proximity of $\mathrm{D}_{0}$. In turn, increasing the van der Waals interaction to an unrealistic strength with on-site potential $V_{V}=-0.5 \mathrm{eV}$ [Fig. 2(e)] renders the replica $\mathrm{D}_{1}$ of the Dirac cone visible near $E=0.114 \mathrm{eV}$ (see Fig. 5) in addition to the main cone $\mathrm{D}_{0}$ at $E=-0.07 \mathrm{eV}$. We note that the main cone $\mathrm{D}_{0}$ shows a finite gap of about $10 \mathrm{meV}$, while the replica cone appears gapless [compare Fig. 5 (a) and (b)]. The DOS in the $B-E$ representation [Fig. 6(a)] and $B-N$ representation [Fig. 6(b)] show now two emerging fans of relativistic dispersion $B \sim E^{2}$ [Fig. 6(a)] and linear dispersion $B \sim N$. The Fermi velocity of the main cone is $v_{F}=0.62 \cdot 10^{6} \mathrm{~m} / \mathrm{s}$, while we find $v_{F}=0.39 \cdot 10^{6} \mathrm{~m} / \mathrm{s}$ for the secondary cone. The calculated density of states [see Fig. 6] shows the presence of Landau levels of Dirac fermions emerging from two Dirac points. The curvature of the parabolas determined by $v_{\mathrm{F}}$ again fits well to the DOS near both $\mathrm{D}_{0}$ and $\mathrm{D}_{1}$ [see red dashed parabolas in Fig. 6(a)]. The additional features emerging near $E=0.03 \mathrm{eV}$ and $E=-0.19 \mathrm{eV}$ at $B=0$ [see arrows in Fig. 6(a)] resulting from the regions of non-relativistic quasi-quadratic dispersion remain present for this much stronger superlattice potential. Thus, the coexistence in the spectrum of a Dirac-like and a Schrödinger-like diamagnetic response persists. As a function of back gate voltage, the satellite structures for relativistic and non-relativistic particles [Fig. 6(b)] are similar. However, as discussed above, they can be well distinguished by the presence of the zeroth Landau level for relativistic dispersion. A more direct approach to observe the two different dispersion relations would be a direct energy dependent measurement. For example, measuring the optical transitions within the satellite structures and within the Landau levels of the main cone ${ }^{35}$ would allow to distinguish between these dispersion relations.

While we do not specifically address disorder in this work, we surmise the satellite structures induced by the 
moiré pattern will respond drastically different to different classes of disorder. Short-range disorder such as lattice vacancies softens all features of the DOS, as numerical tests have shown. However, at realistic disorder concentration the Landau levels and satellites are still well discernable in line with previous studies. ${ }^{25}$ Longrange disorder, on the other hand, may strongly wash out these structures.

\section{CONCLUSION}

We have simulated the electronic structure of a large graphene flake on a hexagonal boron nitride substrate as a function of a perpendicular magnetic field. We have shown that the periodic moiré potential leads to the formation of the Hofstadter butterfly and satellite structures. For a realistic substrate potential, satellites close to the Dirac point feature a parabolic dispersion and cannot be considered replica of the Dirac cone. We have observed that when the $B$ field dependence is measured as a function of the back gate voltage rather than the energy Landau levels for Schrödinger-like and Dirac-like dispersion display the same (approximately) linear behavior. As the unambiguous hallmark for the (non) relativistic response we have identified the (absence) presence of the zeroth Landau level at the satellite and its associated Hofstadter butterfly structure. Our findings thus suggest that the absence of Hofstadter butterfly structure in recent experiments is due to the fact that the observed satellite structure results from parabolic bands rather than replica of the Dirac cone.

\section{ACKNOWLEDGMENTS}

We gratefully acknowledge support from the doctoral colleges CMS (TU Vienna) and Solids4Fun (FWF), as well as by ViCom (SFB 041-ViCom).
* florian.libisch@tuwien.ac.at

1 V. Geringer, M. Liebmann, T. Echtermeyer, S. Runte, M. Schmidt, R. Rückamp, M. C. Lemme, and M. Morgenstern. Phys. Rev. Lett. 102, 076102 (2009).

2 J. Martin, N. Akerman, G. Ulbricht, T. Lohmann, J. H. Smet, K. von Klitzing, and A. Yacoby. Nature Phys., 4, 144 (2007).

3 Castro Neto, F. Guinea, Nmr Peres, Ks Novoselov, and Ak Geim. Rev. Mod. Phys. 81, 109 (2009).

4 E. N. Voloshina, E. Fertitta, A. Garhofer, F. Mittendorfer, M. Fonin, A. Thissen, and Yu Dedkov. Sc. Rep. 3, 2045 (2013).

5 S. Marchini, S. Günther, and J. Wintterlin. Phys. Rev. B 76, 075429 (2007).

${ }^{6}$ Guohong Li, A. Luican, and Eva Y. Andrei. Phys. Rev. Lett. 102, 176804 (2009).

7 C. R. Dean, A. F. Young, I. Meric, C. Lee, L. Wang, S. Sorgenfrei, K. Watanabe, T. Taniguchi, P. Kim, K. L. Shepard, and J. Hone. Nature Nano. 5, 722 (2010).

8 I. Pletikosić, M. Kralj, P. Pervan, R. Brako, J. Coraux, A. T. N'Diaye, C. Busse, and T. Michely. Phys. Rev. Lett. 102, 056808 (2009).

9 Jiamin Xue, Javier Sanchez-Yamagishi, Danny Bulmash, Philippe Jacquod, Aparna Deshpande, K. Watanabe, T. Taniguchi, Pablo Jarillo-Herrero, and Brian J. LeRoy. Nature Mat. 10, 282 (2011).

10 Régis Decker, Yang Wang, Victor W. Brar, William Regan, Hsin-Zon Tsai, Qiong Wu, William Gannett, Alex Zettl, and Michael F. Crommie. Nano Lett., 11, 2291 (2011).

11 Cheol-Hwan Park, Li Yang, Young-Woo Son, Marvin L. Cohen, and Steven G. Louie. Phys. Rev. Lett. 101, 126804 (2008).

12 Matthew Yankowitz, Jiamin Xue, Daniel Cormode, Javier D. Sanchez-Yamagishi, K. Watanabe, T. Taniguchi, Pablo Jarillo-Herrero, Philippe Jacquod, and Brian J. LeRoy. Nature Phys. 8, 382 (2012).

13 D. Subramaniam, F. Libisch, Y. Li, C. Pauly, V. Geringer, R. Reiter, T. Mashoff, M. Liebmann, J. Burgdörfer,
C. Busse, T. Michely, R. Mazzarello, M. Pratzer, and M. Morgenstern. Phys. Rev. Lett. 108, 046801 (2012).

14 Jannik C. Meyer, C. O. Girit, M. F. Crommie, and A. Zettl. App. Phys. Lett. 92, 123110 (2008).

15 R. Bistritzer and A. H. MacDonald. Phys. Rev. B, 84, 035440 (2011).

16 Pilkyung Moon and Mikito Koshino. Phys. Rev. B 88, 241412 (2013).

17 G. L. Yu, et al. Nature Phys. 10, 525 (2014).

18 B. Hunt, J. D. Sanchez-Yamagishi, A. F. Young, M. Yankowitz, B. J. LeRoy, K. Watanabe, T. Taniguchi, P. Moon, M. Koshino, P. Jarillo-Herrero, and R. C. Ashoori. Science 340, 1427 (2013).

19 JI. I. Rabi. Z. f. Physik A 49, 507 (1928).

20 B. Sachs, T. O. Wehling, M. I. Katsnelson, and A. I. Lichtenstein. Phys. Rev. B 84, 195414 (2011).

21 Rafael Martinez-Gordillo, Stephan Roche, Frank Ortmann, and Miguel Pruneda. Phys. Rev. B 89, 161401 (2014).

22 Mikito Koshino and Tsuneya Ando. Phys. Rev. B 73, 155304 (2006).

23 C. R. Dean, L. Wang, P. Maher, C. Forsythe, F. Ghahari, Y. Gao, J. Katoch, M. Ishigami, P. Moon, M. Koshino, T. Taniguchi, K. Watanabe, K. L. Shepard, J. Hone, and P. Kim. Nature 497, 598 (2013).

24 Douglas R. Hofstadter. Phys. Rev. B, 14, 2239 (1976).

25 F. Libisch, S. Rotter, J. Güttinger, C. Stampfer, and J. Burgdörfer. Phys. Rev. B 81, 245411 (2010).

26 S. Das Sarma, Shaffique Adam, E. H. Hwang, and Enrico Rossi. Electronic transport in two-dimensional graphene. Rev. Mod. Phys. 83, 407 (2011).

27 M.V. Berry and R.J. Mondragon. Proc. R. Soc. Lond., Ser. A 412, 53 (1987).

28 A. F. Young, C. R. Dean, L. Wang, H. Ren, P. CaddenZimansky, K. Watanabe, T. Taniguchi, J. Hone, K. L. Shepard, and P. Kim. Nature Phys. 8, 550 (2012).

29 L.D. Landau and E.M. Lifshitz. Quantum Mechanics. Nonrelativistic theory, volume 3. Pergamon Press, London, 2nd 
edition (1965).

30 R. Reiter, U. Derra, S. Birner, B. Terrés, F. Libisch, J. Burgdörfer, and C. Stampfer. Phys. Rev. B 89, 115406 (2014).

31 M. C. Geisler, J. H. Smet, V. Umansky, K. von Klitzing, B. Naundorf, R. Ketzmerick, and H. Schweizer. Phys. Rev. Lett. 92, 256801 (2004).

32 R. E. Peierls. Z. Phys., 80, 763 (1933).
33 S. Reich, J. Maultzsch, C. Thomsen, and P. Ordejón. Phys. Rev. B 66, 035412 (2002).

34 Cheol-Hwan Park, Li Yang, Young-Woo Son, Marvin L. Cohen, and Steven G. Louie. Nature Phys. 4, 213 (2008).

35 Z.-G. Chen, Z. Shi, W. Yang, X. Lu, Y. Lai, H. Yan, F. Wang, G. Zhang, and Z. Li, Nature Comm. 5, 4416 (2014). 